\title{
Atypical Presentation of Cardiac Tamponade as a Cause of Acute Liver Injury: Case Report and Review of Literature
}

\author{
Sarang Thaker ${ }^{1}$, Cemal Yazici ${ }^{2}$, Sean Koppe ${ }^{2}$ \\ 1. Department of Medicine, University of Illinois at Chicago, Chicago, USA 2. Department of Medicine, Division of \\ Gastroenterology and Hepatology, University of Illinois at Chicago, Chicago, USA
}

Corresponding author: Sarang Thaker, sthake2@uic.edu

\begin{abstract}
Pericardial tamponade is a rare cause of acute liver injury due to the compressive effects of an effusion resulting in a poor cardiac output which ultimately leads to ischemia-induced injury. We present a patient with chronic hepatitis $C$ infection and end-stage renal disease who was transferred to our center for further evaluation and management of acute liver injury after presenting to an outside hospital with left upper quadrant abdominal pain, nausea and vomiting. The patient was discovered to have tamponade physiology on transthoracic echocardiogram as an underlying cause of his acute liver injury despite lack of clinical tamponade features. He required pericardiocentesis which eventually led to resolution of the acute liver injury and he was discharged home on day twelve after full recovery. We review the existing literature regarding the epidemiology, pathophysiology, clinical presentation, diagnosis, and treatment of ischemic hepatitis, which is associated with high mortality; therefore early recognition and treatment of the underlying cause are paramount.
\end{abstract}

Categories: Internal Medicine, Gastroenterology

Keywords: acute liver injury, cardiac tamponade, ischemic hepatitis, shock liver

\section{Introduction And Background}

Ischemic hepatitis is a rare cause of acute liver injury (ALI) and is associated with various etiologies including acute cardiac failure, trauma, hemorrhage, and respiratory failure that all result in poor perfusion and oxygen delivery to the liver [1]. Patients present with elevated transaminases, often to the thousands, typically occurring after a period of hypotension; however, cases may also occur without a hypotensive episode. In fact, up to half of the cases of "shock liver" have been noted to occur in patients without shock [1]. Serum transaminases typically return to baseline level within 10 days after treating the underlying cause [2]. Patients may present with symptoms suggestive of acute hepatitis, including nausea, vomiting, abdominal pain, and malaise [3]. We present a rare case of ALI caused by pericardial tamponade in a patient with hepatitis C infection (HCV) and end-stage renal disease (ESRD) which required pericardiocentesis, ultimately leading to resolution of ALI. We also present an overview of the current literature with regards to

Received 05/21/2018 Review began $05 / 31 / 2018$ Review ended 06/08/2018 Published 06/11/2018

\section{๑) Copyright 2018}

Thaker et al. This is an open access article distributed under the terms of the Creative Commons Attribution License CC-BY 3.0., which permits unrestricted use, distribution, and reproduction in any medium, provided the original author and source are credited. this entity.

\section{Case}

A 56-year-old male with a past medical history of HCV, ESRD on hemodialysis, hypertension, and diabetes mellitus presented to an outside hospital with left-sided abdominal pain associated with nausea and vomiting after completion of hemodialysis earlier that day. Laboratory studies revealed elevated liver enzymes (Aspartate transaminase (AST) of $4200 \mathrm{U} / \mathrm{L}$, alanine transaminase (ALT) of $3600 \mathrm{U} / \mathrm{L}$, total bilirubin of $2.7 \mathrm{mg} / \mathrm{dl}$ ) and coagulopathy (International normalized ratio (INR) of 2.9) along with severe lactic acidosis to $17 \mathrm{mmol} / \mathrm{L}$. The patient was subsequently transferred to our center for evaluation and management of ALI.

Upon arrival, the patient was hypothermic to $35.7^{\circ} \mathrm{C}$, but otherwise hemodynamically stable and reported resolved abdominal pain. Physical exam was remarkable for hepatomegaly and negative for distended neck veins or muffled heart sounds. Electrocardiogram (ECG) did not demonstrate low voltages.

ALI workup was initiated and abdominal ultrasonography demonstrated a normal liver with patent hepatic vasculature and a normal gallbladder without stones intrahepatic ductal dilatation, but it did note a pericardial effusion. Given these findings, an echocardiogram was performed and revealed a $2.0 \mathrm{~cm}$ circumferential pericardial effusion, flattening of the interventricular septum during inspiration, and a dilated inferior vena cava to $2.8 \mathrm{~cm}$ consistent with tamponade physiology (Figure 1) without the clinical features of tamponade. Computed tomography (CT) also revealed the presence of pericardial fluid accumulation (Figure 2). On hospital day two, the patient underwent pericardiocentesis with the removal of $500 \mathrm{ml}$ of bloody fluid and drain placement. The patient improved clinically with decreasing transaminases (AST $1549 \mathrm{U} / \mathrm{L}$ and ALT $1644 \mathrm{U} / \mathrm{L}$ at 12 hours after intervention). 


\section{Cureus}

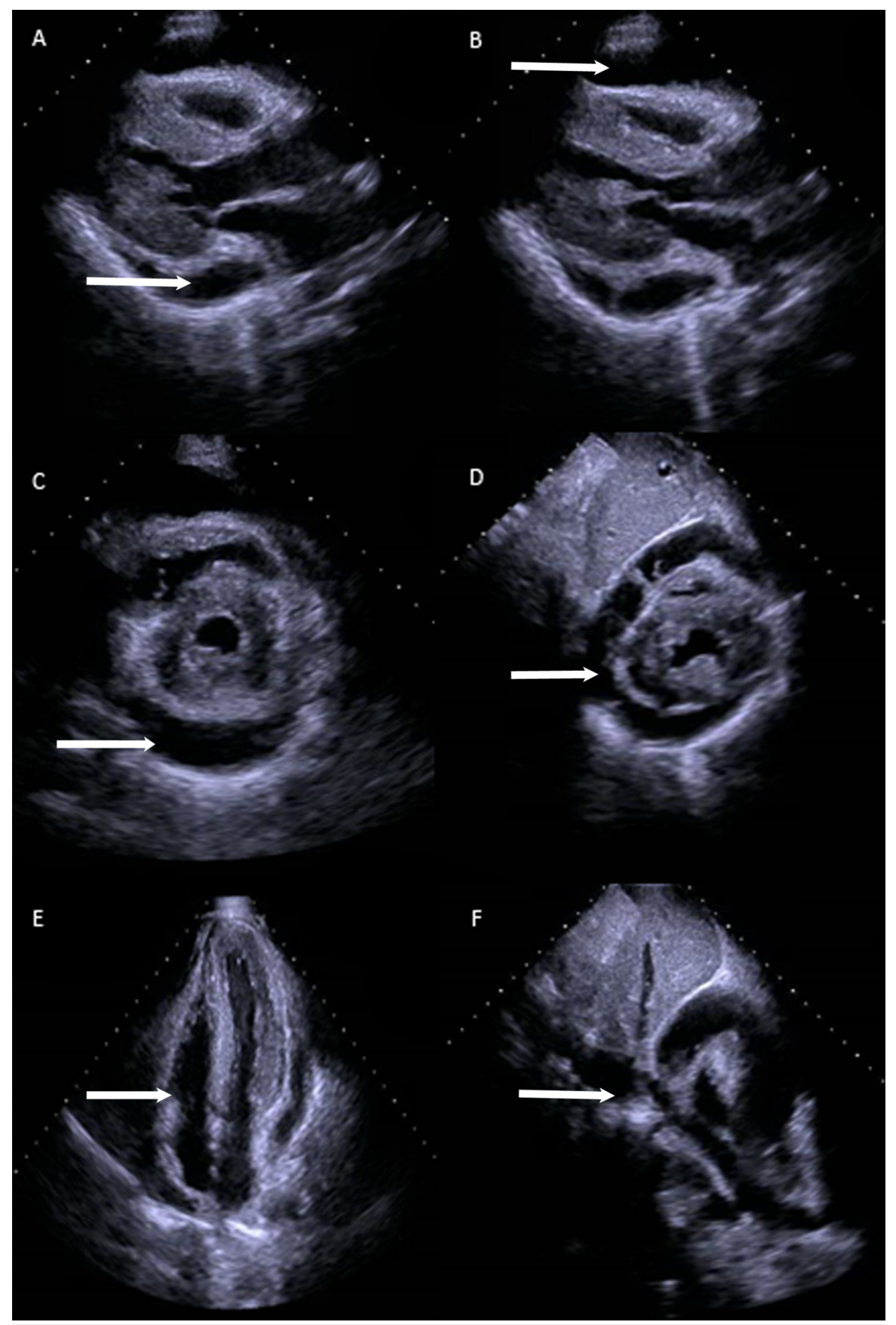

\section{FIGURE 1: Echocardiogram.}

Echocardiographic findings demonstrating pericardial effusion in parasternal long view (A, B) and short axis view $(C, D)$, flattening of interventricular septum and right ventricular collapse $(E)$, dilated Inferior Vena Cava (F) as indicated by arrows. 


\section{Cureus}

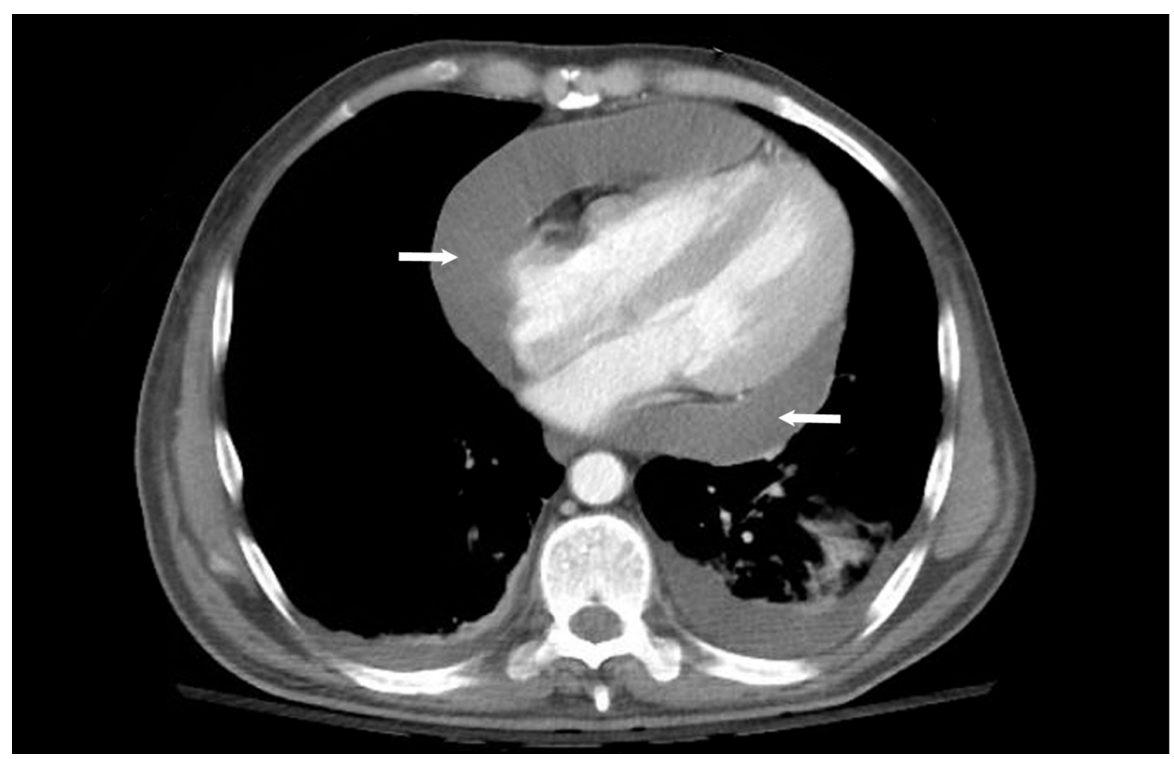

FIGURE 2: Computed tomography.

Computed tomography portraying circumferential pericardial effusion (arrows).

Later that evening, the patient's mental status acutely worsened coinciding with hypotension and tachycardia, ultimately requiring intubation. Shortly thereafter he sustained pulseless electrical activity (PEA) arrest twice with successful return of spontaneous circulation after two and five rounds of cardiopulmonary resuscitation, respectively. Telemetry did not reveal an antecedent arrhythmia. Bedside echocardiogram did not demonstrate tamponade physiology; however, left ventricular collapse was noted indicative of volume depletion. Laboratory findings revealed a hemoglobin of $3.9 \mathrm{~g} / \mathrm{dL}$ and INR of 4.9; CT head was negative for acute hemorrhage, but CT chest/abdomen/pelvis revealed hemoperitoneum and subcapsular hematoma likely secondary to pericardiocentesis. The patient was aggressively resuscitated with blood products and fluids with appropriate response and was successfully weaned off vasopressors. He was in disseminated intravascular coagulopathy (DIC) and required fresh frozen plasma, cryoprecipitate, and platelet transfusions. The patient underwent emergent right internal mammary artery embolization as it was noted that the pericardial drain coursed adjacent to this vessel. Troponinemia developed and, on postpericardiocentesis day two, peaked at a level of $49 \mathrm{ng} / \mathrm{ml}$ with no ST elevations on electrocardiogram, deemed to be a non-ST elevation myocardial infarction (NSTEMI); however, the patient was a poor candidate for both medical and percutaneous interventions given coagulopathy and acute bleed.

Echocardiogram on hospital day four noted normal left ventricular function with regional wall motion abnormalities and reduced right ventricular function. The pericardial drain was removed, the patient was successfully extubated, DIC resolved, coagulopathy improved, and transaminases further decreased as shown in Figure 3. The patient was transferred out of the Intensive Care Unit (ICU) and the remainder of his hospital course was uncomplicated. He was safely discharged home within one week. Laboratory studies at the time of discharge were remarkable for an AST of $63 \mathrm{U} / \mathrm{L}$, ALT of $161 \mathrm{U} / \mathrm{L}$, INR of 1.1, and hemoglobin of $10.8 \mathrm{gm} / \mathrm{dl}$. 


\section{Cureus}

Transaminase Levels Throughout Hospitalization

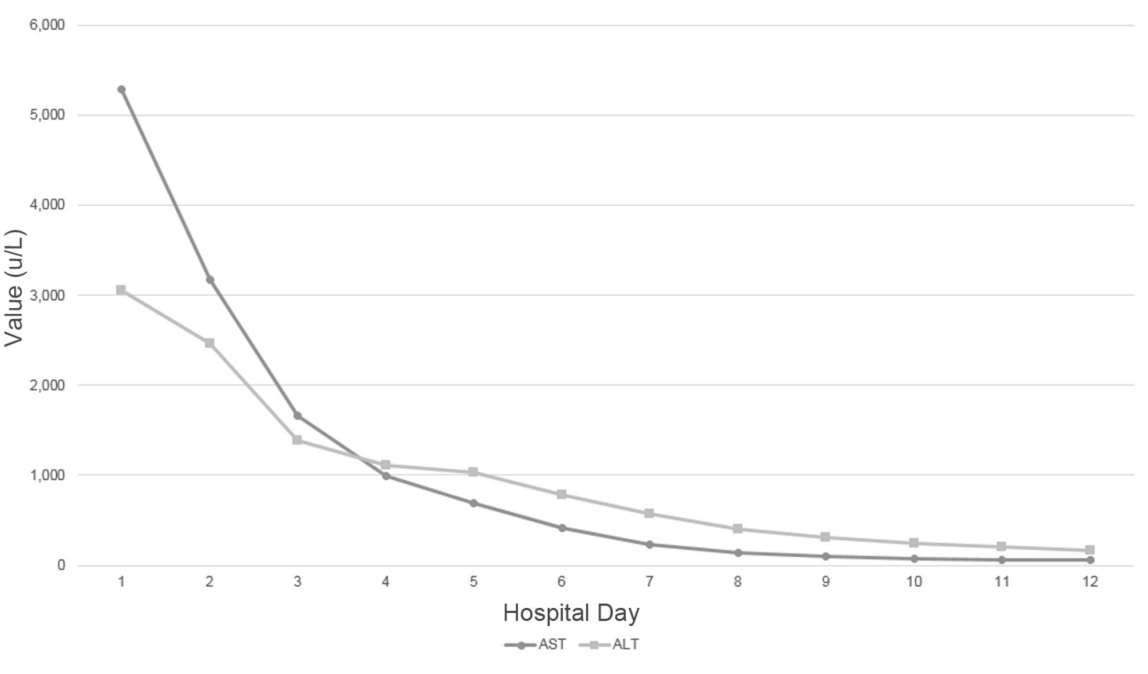

FIGURE 3: Transaminase trend.

Trend of transaminases throughout hospitalization; pericardiocentesis performed on hospital day two.

The etiology of this patient's pericardial effusion remains unclear. Cytology and anaerobic, aerobic, fungal, and acid-fast bacilli cultures were negative. Blood urea nitrogen was not significantly elevated and thus less likely the cause. Infectious workup was negative for varicella, cytomegalovirus, Epstein-Barr virus, coxsackievirus, echovirus, adenovirus, human immunodeficiency virus, tuberculosis quantiferon, histoplasmosis, and blastomycosis. Autoimmune studies including antinuclear antibody, anti-neutrophil cytoplasmic antibody, anti-mitochondrial antibody, anti-smooth muscle antibody, anticardiolipin, and anti$\beta 2$-glycoprotein were unremarkable.

\section{Review}

\section{Epidemiology of ischemic hepatitis}

The incidence of ischemic hepatitis varies and was found to be $0.16 \%$ for general medicine ward, $0.9 \%$ for ICU and $2.6 \%$ for cardiac ICU admissions $[1,4]$. The liver is generally well-protected from ischemia due to its dual blood supply from the portal vein and hepatic artery [4]. A meta-analysis including 1782 subjects from 24 studies revealed similar results [5]. Ischemic hepatitis was seen in $0.2 \%$ of all hospital admissions (both general medicine and intensive care). Ischemic hepatitis was also seen in $2.5 \%$ of ICU and $40 \%$ of all admissions in which patients had transaminase levels > 10 times the upper limit of normal. A recent singlecenter study noted ischemic hepatitis in $4 \%$ of ICU admissions [6].

\section{Clinical presentation}

Patients with ischemic hepatitis present with a variety of symptoms such as weakness, dyspnea, and abdominal pain. Transaminase levels increase within 48 hours after the inciting event and coagulopathy with an elevated INR subsequently occurs [4]. Bilirubin may increase as well, but jaundice is uncommon. Patients with ischemic hepatitis secondary to cardiac tamponade typically have evident signs and symptoms of tamponade on presentation. This particular patient had no evidence of cardiac compromise such as hypotension, distended neck veins, diminished cardiac sounds upon his presentation which highlights the rarity of this case. In the absence of telltale signs and symptoms of tamponade, clinicians must be suspicious of tamponade when evaluating a patient for ischemic hepatitis. Several cases of tamponade resulting in ALI have been reported, yet these patients either presented with signs and symptoms of outright tamponade or had malignancy resulting in pericardial effusion, recent cardiothoracic surgery, or Mycobacterium tuberculosis infection, our case had none of these [7-11]. Cardiac tamponade is a clinical diagnosis. Electrocardiogram typically demonstrates low voltages or electrical alternans which is fairly specific for tamponade; again, the patient presented above lacked these typical findings on ECG [12, 13]. Echocardiography is recommended to assess for hemodynamic compromise secondary to pericardial effusions and may reveal chamber collapse, especially right-sided, suggestive of elevated intrapericardial pressures [12]. A highly specific and sensitive finding consistent with tamponade physiology is a reversal of the normal respiratory variations of flow and volume of the ventricles in addition to a dilated inferior vena cava, which the patient in this case displayed $[14,15]$. The diagnosis of tamponade in this patient was confirmed as pericardiocentesis resulted in clinical improvement as well as hemodynamic stability.

\section{Etiology and pathophysiology}

Predisposing factors include many entities, particularly cardiovascular disease, sepsis, and chronic 
respiratory disease. The most common cause of ischemic hepatitis was noted to be cardiac, including arrhythmias, myocardial infarction, and heart failure $[1,16]$. Seeto et al. studied the pathophysiology of ischemic hepatitis in a cohort of 31 patients with ischemic hepatitis matched to 31 control patients with both groups consisting of patients with systolic blood pressures $<75 \mathrm{mmHg}$ for at least 15 minutes. The group concluded that hypotension alone did not lead to ischemic hepatitis and noted that each patient that developed ischemic hepatitis had underlying cardiac disease, most commonly right-sided heart failure, which caused hepatic congestion. Indeed, as cardiac output declines or right heart pressures increase, the pressures required to perfuse the hepatic sinusoids increase resulting in reduced portal blood flow [17]. Birrer et al. and Seeto et al. concluded that transient hypotension in patients with underlying hepatic congestion due to right heart failure is sufficient to cause notable hepatocellular necrosis [18, 19]. In a recent study, Van den broecke et al. identified 1116 cases of ischemic hepatitis in a single-center ICU and determined that approximately half of these cases were as a result of cardiac failure [6]. Our patient had preexistent liver disease secondary to hepatitis $\mathrm{C}$ which made him relatively more vulnerable to developing ischemic hepatitis from hepatic congestion due to his pericardial effusion despite lacking overt signs of tamponade. In fact, Waseem et al. determined that patients with baseline liver disease have increased risk of developing acute liver injury in the setting of unstable hemodynamics [20]. This patient also had a history of hypertension which has been linked to diastolic dysfunction, providing another predisposing risk factor. He also has ESRD which has also been linked to the development of hepatic congestion [21]. Our patient emphasizes the role of passive hepatic congestion in the pathophysiology of ischemic hepatitis, especially when considering that his aminotransferases improved after pericardiocentesis and continued to decline even after PEA arrest and hemorrhagic shock.

In heart failure, decreased cardiac output causes a compensatory increase in systemic vascular resistance (SVR). The splanchnic vessels constrict disproportionately more than systemic vessels resulting in reduced perfusion to the liver [4]. Henrion et al. retrospectively studied the hemodynamics in 142 patients with ischemic hepatitis due to heart failure, chronic respiratory failure, and shock. This study revealed that cardiac compromise leads to liver ischemia, respiratory compromise causes hypoxemia resulting in hepatic injury, and septic and hypovolemic shock lead to an inability for the body to meet hepatic oxygenation demands [1]. Saner et al. performed a retrospective observational cohort study on 13 patients with cardiogenic shock and ALI. The group determined that patients who survived had a significantly higher cardiac index upon presentation compared to patients who did not survive, and recovery of cardiac index was associated with improved hepatic function [22]. The group noticed elevated post-shock central venous pressures (CVP) in the survival group and that hepatic function recovered despite this finding, indicating that cardiac index has more of an effect on hepatic congestion than CVP alone [22]. In a meta-analysis that included 1782 ALI cases, Tapper et al. showed that $78.2 \%$ of subjects had an inciting cardiac event, $52.9 \%$ had a hypotensive insult and $23.4 \%$ had sepsis [5].

Regarding patients with chronic respiratory failure, progressive hypoxemia causes hepatic injury, usually without an inciting event [4]. Van den broecke et al. noted that approximately $10 \%$ of ischemic hepatitis cases in a single-center ICU were related to either acute respiratory failure or acute-on-chronic respiratory failure [6]. Henrion et al. examined cases of ischemic hepatitis due to chronic respiratory failure without left-sided heart failure in order to determine if hypoxemia independently causes hepatic injury. The cohort of patients included those with restrictive and obstructive lung disease. The degree to which hypoxemia caused liver injury was confounded by systemic hypotension and elevated CVP, hence the group performed hemodynamic studies. When comparing hemodynamics between this cohort against a cohort with ischemic hepatitis due to congestive heart failure, the group revealed that the systemic hypotension in the respiratory failure group was due to reduced SVR and these patients had an increased cardiac index, whereas the heart failure group had systemic hypotension due to reduced cardiac index. When measuring oxygen delivery, the chronic respiratory failure group had profoundly low oxygen delivery due to severe hypoxemia (pO2 < 60 $\mathrm{mmHg}$ ) [23]. The group concluded that hepatic injury in chronic respiratory failure patients is due to extreme hypoxemia primarily from progression of severe pulmonary disease and not ischemic injury due to hypotension [23]. Patients with obstructive sleep apnea or obesity hypoventilation syndrome may also develop ischemic hepatitis due to extreme hypoxemia [24]. Additionally, these patients may also have rightsided heart failure from pulmonary hypertension with subsequent hepatic congestion.

In septic shock, hepatocytes have an increased oxygen demand coupled with a reduced ability to extract oxygen resulting in injury. Van den broecke et al. noted septic shock as the cause of ischemic hepatitis in roughly $30 \%$ of ICU admissions [6]. Endotoxin stimulates Kupffer cells and causes an inflammation cascade in the parenchyma [4]. Mesenteric blood flow may be decreased due to vasoconstriction and shunting of blood elsewhere or increased due to endotoxin-mediated vasodilation. Henrion et al. noted that cardiac index was often increased due to reduced SVR, and oxygen delivery was maintained. They noted reduced intrahepatic oxygen tension indicating an increased oxygen demand and decreased extraction, resulting in inflammation and injury [1]. In fact, altered hepatic perfusion results in the release of pro-inflammatory cytokines such as tumor necrosis $\alpha$ and interleukin- 8 resulting in dysregulated metabolism, immune response, and toxin clearance, exacerbating the underlying sepsis [25]. Activated neutrophils worsen liver injury by adhering to hepatic endothelial cells and generating reactive oxygen species [26]. Therefore, ischemic hepatitis in sepsis is primarily due to the reduced hepatic extraction of oxygen and not ischemia [1].

Other causes of ischemic hepatitis have been identified and include severe anemia where patients present with extremely low hemoglobin levels and severe lactic acidosis indicating poor tissue oxygenation. In a case report of ALI due to a hemoglobin of $1.8 \mathrm{~g} / \mathrm{dl}$, the authors postulated that anemia to this degree decreased 
hepatic oxygenation and resulted in acute liver injury [27]. Van den broecke et al. observed hypovolemic shock as a cause of nearly $10 \%$ of ischemic hepatitis cases in an ICU setting [6]. An additional case report noted the development of ischemic hepatitis due to septic emboli from bacterial endocarditis [28]. Cocaineinduced hepatic injury is dose-dependent and is related to P450 activity on cocaine metabolites generating oxidative stress. Cocaine also causes hepatic vasoconstrictive effect leading to hepatic injury [29]. Additionally, carbon monoxide poisoning has been shown to result in severe ischemic hepatitis due to reduced oxygen delivery to hepatocytes. In a case of carbon monoxide poisoning, resolution of the liver injury was attained with the treatment of the carboxyhemoglobinemia [30].

\section{Diagnosis}

Ischemic hepatitis is typically diagnosed by laboratory studies demonstrating an acute hypertransaminasemia, usually > 100 times the upper limit of normal [31]. Differential diagnosis includes viral and autoimmune hepatitis, direct liver trauma, vascular disease, toxin-induced such as acetaminophen ingestion, and biliary obstruction secondary to lithiasis. Liver biopsy is rarely performed and usually reveals centrilobular necrosis $[4,7]$. Pathological changes related to ischemic hepatitis are usually observed in zone III near the central vein [4]. The first step in the evaluation of elevated aminotransferases is to obtain abdominal ultrasonography to exclude choledocholithiasis especially in patients with elevated bilirubin, abdominal pain, and signs concerning for infection such as our patient. Next, the pattern of aminotransferase can indicate the etiology. Our patient had an AST > ALT which would normally suggest alcohol ingestion, however the degree of elevation seen in ischemic hepatitis is much greater than that of alcoholic hepatitis which does not exceed $>10$ times the upper limit of normal, substantiating the diagnosis of ischemic hepatitis especially in setting of a normal ultrasound [32]. The case above highlights the complexity of ischemic hepatitis etiologies and emphasizes the necessity of clinical suspicion and obtaining prompt echocardiography in patients with an otherwise unremarkable evaluation.

\section{Prognosis and treatment}

In a retrospective study of 142 ICU patients, 30-day mortality rate of ischemic hepatitis was $52.8 \%$ and oneyear survival rate was $28.3 \%$ with the caveat that this patient population either had decompensated heart failure, exacerbation of chronic respiratory failure, or circulatory shock [1]. Birrer et al. noted a mortality rate of $45 \%$ in 293 cases and death occurred due to sudden cardiorespiratory failure [19]. In a meta-analysis with 1782 cases, the mortality rate of ischemic hepatitis was noted to be approximately 50\% [5]. In a singlecenter cohort of 1116 ICU patients with ischemic hepatitis, Van den broecke et al. observed a $45.0 \%$ mortality rate at 28 days with $18.5 \%$ of cases dying within 24 hours of peak AST level [6]. This study also differentiated mortality rates based on etiology of ischemic hepatitis. The septic shock subgroup and cardiac failure subgroup had a 28 -day mortality rate of $52.9 \%$ and $41.4 \%$, respectively [6]. Fuhrmann et al. showed a statistically significant higher peak of aminotransferases, INR, and lactate was observed in patients who died [33]. Clearly, there is a significant mortality risk in ischemic hepatitis and early identification of underlying etiology and its timely treatment is key for survival as demonstrated by this case.

Treatment of ischemic hepatitis is targeted towards correcting the underlying etiology. In patients who develop ALI secondary to cardiac tamponade, pericardiocentesis should be performed and the resultant resolution of transaminitis is typically seen within 72 hours. Additionally, correction of pathology that leads to the formation of pericardial effusion is recommended. Elevated liver enzymes in patients with ischemic hepatitis typically undergo a $50 \%$ reduction within 72 hours of correcting the underlying cause [4].

Patients with heart failure and elevated CVP may require inotropic-supported diuresis to decrease hepatic congestion and increase splanchnic blood flow [5]. Septic shock patients benefit from vasopressors, antimicrobials, fluid resuscitation, and supportive care [5]. Hepatobiliary transport is reduced in sepsis leading to cholestasis. Resultant translocation of endotoxin and endotoxin-mediated response further worsens sepsis as detailed earlier [26]. Antimicrobial selection should aim to treat intra-abdominal organisms such as Gram-negative rods and anaerobes in septic patients with findings and clinical signs of intra-abdominal source.

In patients with chronic respiratory failure, treatment should be directed towards correcting hypoxemia as well as treating the underlying cause with supplemental oxygen, possible ventilatory support, nebulized beta-agonists and anticholinergics, corticosteroids, and possibly antimicrobials [34]. As noted above, both restrictive and obstructive lung disease patients can develop ischemic hepatitis; therefore, the etiology of chronic respiratory failure should be managed with its respective treatment [23]. Patients with obstructive sleep apnea should be treated with non-invasive positive pressure ventilation to minimize nocturnal oxygen desaturation [24]. Finally, in patients with right-sided heart failure due to pulmonary hypertension, treatment must emphasize improving hemodynamics and addressing the etiology of pulmonary hypertension [35].

Ischemic hepatitis due to toxins such as cocaine and 3,4-methylenedioxymethamphetamine (MDMA) ingestion is largely treated with supportive care as they present with a constellation of symptoms including, but not limited to, hyperthermia, rhabdomyolysis, hypotension, and DIC [29]. As discussed earlier, roughly one-half of patients with acute liver injury due to ischemic hepatitis do not survive to discharge, therefore it is paramount that early recognition and diagnosis be made in order to expedite treatment [19]. 


\section{Conclusions}

This case emphasizes the requirement for proper workup of any patient presenting with the acute liver injury. As various studies have explained, the most common causes of elevated aminotransferases to the thousands include toxins, trauma, acute viral hepatitis, and ischemic hepatitis which is most often cardiac in origin due to arrhythmia, myocardial infarction, heart failure, or tamponade as in this patient. Though this patient did not exhibit the classic signs and symptoms of pericardial tamponade, it nonetheless was the cause of the presenting ALI. Furthermore, as the review of the literature has demonstrated, ischemic hepatitis is prevalent and associated with high mortality, underscoring the necessity for vigilance and early recognition in order to improve outcomes.

\section{Additional Information \\ Disclosures}

Conflicts of interest: In compliance with the ICMJE uniform disclosure form, all authors declare the following: Payment/services info: All authors have declared that no financial support was received from any organization for the submitted work. Financial relationships: All authors have declared that they have no financial relationships at present or within the previous three years with any organizations that might have an interest in the submitted work. Other relationships: All authors have declared that there are no other relationships or activities that could appear to have influenced the submitted work.

\section{References}

1. Henrion J, Schapira M, Luwaert R, Colin L, Delannoy A, Heller FR: Hypoxic hepatitis: clinical and hemodynamic study in 142 consecutive cases. Medicine. 2003, 82:392-406. Accessed: January 17, 2017: https://journals.lww.com/mdjournal/Fulltext/2003/11000/Hypoxic_Hepatitis_Clinical_and_Hemodynamic_Study.3.aspx. 10.1097/01.md.0000101573.54295.bd

2. Gitlin N, Serio K: Ischemic hepatitis: widening horizons . Am J Gastroenterol. 1992, 87:831-836. Accessed: January 17, 2017: https://www.ncbi.nlm.nih.gov/pubmed/1615936.

3. Bynum TE, Boitnott JK, Maddrey WC: Ischemic hepatitis. Digest Dis Sci. 1979, 24:129-135. Accessed: January 17, 2017: https://link.springer.com/article/10.1007/BF01324740. 10.1007/bf01324740

4. Ebert EC: Hypoxic liver injury. Mayo Clin Proc. 2006, 81:1232-1236. Accessed: January 17, 2017: https://www.mayoclinicproceedings.org/article/S0025-6196(11)61235-4/abstract. 10.4065/81.9.1232

5. Tapper EB, Sengupta N, Bonder A: The incidence and outcomes of ischemic hepatitis: a systematic review with meta-analysis. Am J Med. 2015, 128:1314-1321. Accessed: January 17, 2017 : https://www.ncbi.nlm.nih.gov/pubmed/26299319. 10.1016/j.amjmed.2015.07.033

6. Van den broecke A, Van Coile L, Decruyenaere A, Colpaert K, Benoit D, Van Vlierberghe H, Decruyenaere J: Epidemiology, causes, evolution and outcome in a single-center cohort of 1116 critically ill patients with hypoxic hepatitis. Ann Intensive Care. 2018, 8:15. Accessed: March 24, 2018: https://www.ncbi.nlm.nih.gov/pmc/articles/PMC5790763/. 10.1186/s13613-018-0356-z

7. López-Méndez E, López-Méndez I, Hernández-Reyes P, et al.: A 57 year old man with chronic renal failure and cardiac tamponade who developed ischemic hepatitis. Ann Hepatol. 2006, 5:50-52. Accessed: February 2, 2017: https://www.ncbi.nlm.nih.gov/pubmed/16531967.

8. Delgado C, Barturen F: Atrial tamponade causing acute ischemic hepatic injury after cardiac surgery . Clin Cardiol. 1999, 22:242-244. Accessed: February 2, 2017: https:/www.ncbi.nlm.nih.gov/pubmed? term=atrial\%20tamponade\%20causing\%20acute\%20ischemic\%20hepatic\%20injury\%20artery\%.... $10.1002 /$ clc.4960220319

9. Leditschke IA, Fraser JF, Sommerville RS, Atherton J: Acute hepatic failure caused by an acute aortic dissection with cardiac tamponade: a case report. Crit Care Resusc. 2004, 6:105-108. Accessed: February 2 , 2017: https://www.ncbi.nlm.nih.gov/pubmed/? term=Acute+hepatic+failure+caused+by+an+acute+aortic+dissection+with+cardiac+tam....

10. Boendermaker AE, Boumans D, van Zanten RAA, Idzerda H, van de Hout H, Veneman Th F: Elevated liver enzymes and renal failure, with a surprising outcome. two similar cases. Neth J Crit Care. 2013, 17:33-36. Accessed: February 2, 2017: https://nvic.nl/elevated-liver-enzymes-and-renal-failure-surprising-outcometwo-similar-cases.

11. Bollela VR, Puga FG, Carvalho R: Tuberculous cardiac tamponade presenting as severe hypoxic hepatitis. J Health Soc Sci. 2016, 1:279-286. Accessed: February 2, 2017: https://www.researchgate.net/publication/312500166_Tuberculous_cardiac_tamponade_presenting_as_severe_hypoxic_hepat 10.19204/2016/tbrc28

12. Spodick DH: Acute cardiac tamponade. N Engl J Med. 2003, 349:684-690. Accessed: March 24, 2018: https://www.nejm.org/doi/full/10.1056/NEJMra022643. 10.1056/NEJMra022643

13. Bruch C, Schmermund A, Dagres N, Bartel T, Caspari G, Sack S, Erbel R: Changes in QRS voltage in cardiac tamponade and pericardial effusion: reversibility after pericardiocentesis and after anti-inflammatory drug treatment. J Am Coll Cardiol. 2001, 38:219-226. Accessed: March 24, 2018: http://www.onlinejacc.org/content/38/1/219. 10.1016/S0735-1097(01)01313-4

14. Himelman RB, Kircher B, Rockey DC, Schiller NB: Inferior vena cava plethora with blunted respiratory response: a sensitive echocardiography sign of cardiac tamponade. J Am Coll Cardiol. 1988, 12:1470-1477. Accessed: March 24, 2018: http://www.onlinejacc.org/content/12/6/1470. 10.1016/S0735-1097(88)80011-1

15. Merce J, Sagrista-Sauleda J, Permanyer-Miralda G, Evangelista A, Soler-Soler J: Correlation between clinical and Doppler echocardiographic findings in patients with moderate and large pericardial effusion: implications for the diagnosis of cardiac tamponade. Am Heart J. 1999, 138:759-764. Accessed: March 24, 2018: https://www.ahjonline.com/article/S0002-8703(99)70193-6/abstract. 10.1016/S0002-8703(99)70193-6

16. Khan FY, Baagar KA: Ischaemic hepatitis precipitated by recurrent episodes of atrial fibrillation . Arab J Gastroenterol. 2013, 14:176-179. Accessed: May 10, 2017: 
https://www.sciencedirect.com/science/article/pii/S1687197913001329. 10.1016/j.ajg.2013.10.002

17. Hosoki T, Arisawa J, Marukawa T, Tokunaga K, Kuroda C, Kozuka T, Nakano S: Portal blood flow in congestive heart failure: pulsed duplex sonographic findings. Radiology. 1990, 174:733-736. Accessed: March 24, 2018: https://pubs.rsna.org/doi/abs/10.1148/radiology.174.3.2406781.

10.1148/radiology.174.3.2406781

18. Seeto RK, Fenn B, Rockey DC: Ischemic hepatitis: clinical presentation and pathogenesis . Am J Med. 2000, 109:109-113. Accessed: January 17, 2017: https://www.amjmed.com/article/S0002-9343(00)004617/abstract. 10.1016/S0002-9343(00)00461-7

19. Birrer R, Takuda Y, Takara T: Hypoxic hepatopathy: pathophysiology and prognosis. Intern Med. 2007, 46:1063-1070. Accessed: February 2, 2017: https://www.ncbi.nlm.nih.gov/pubmed/17634701. 10.2169/internalmedicine.46.0059

20. Waseem N, Limketkai BN, Kim B, Woreta T, Gurakar A, Chen PH: Risk and prognosis of acute liver injury among hospitalized patients with hemodynamic instability: a nationwide analysis. Ann Hepatol. 2018, 17:119-124. Accessed: March 24, 2018:

https://annalsofhepatology.publisherspanel.com/resources/html/article/details?id=158121 \&language=en. 10.5604/01.3001.0010.7543

21. Pahl MV, Vaziri N, Dure-Smith B, Miller R, Mirahmadi MK: Hepatobiliary pathology in hemodialysis patients: an autopsy study of 78 cases. Am J Gastroenterol. 1986, 81:783-787. Accessed: March 24, 2018: https://www.ncbi.nlm.nih.gov/pubmed/3752041.

22. Saner FH, Heuer M, Meyer M, et al.: When the heart kills the liver: acute liver failure in congestive heart failure. Eur J Med Res. 2009, 14:541-546. Accessed: February 2, 2017:

https://www.ncbi.nlm.nih.gov/pmc/articles/PMC3351940/. 10.1186/2047-783X-14-12-541

23. Henrion J, Minette P, Colin L, Schapira M, Delannoy A, Heller FR: Hypoxic hepatitis caused by acute exacerbation of chronic respiratory failure: a case-controlled, hemodynamic study of 17 consecutive cases. Hepatology. 1999, 29:427-433. Accessed: May 10, 2017: https://aasldpubs.onlinelibrary.wiley.com/doi/abs/10.1002/hep.510290202. 10.1002/hep.510290202

24. Mathurin P, Durand F, Ganne N, et al.: Ischemic hepatitis due to obstructive sleep apnea. Gastroenterology. 1995, 109:1682-1684. Accessed: September 24, 2017: https://www.ncbi.nlm.nih.gov/pubmed/7557154. 10.1016/0016-5085(95)90659-2

25. Yan J, Li S, Li S: The role of the liver in sepsis . Int Rev Immunol. 2014, 33:498-510. Accessed: May 10, 2017: https://www.ncbi.nlm.nih.gov/pubmed/24611785. 10.3109/08830185.2014.889129

26. Soultati A, Dourakis SP: Liver dysfunction in the intensive care unit. Ann Gastroenterol. 2005, 18:35-45. Accessed: May 10, 2017: http://www.annalsgastro.gr/index.php/annalsgastro/article/view/304.

27. Okas A, Kowalczyk J, Stein R, Lee D, Berkelhammer C: Hypoxic hepatitis related to profound anemia: how low can you go?. Am J Gastroenterol. 2001, 96:3445-3447. Accessed: May 10, 2017: https://www.ncbi.nlm.nih.gov/pubmed/11774972. 10.1111/j.1572-0241.2001.05381.x

28. Henrich WL, Huehnegarth RJ, Rösch J, Melnyk CS: Gallbladder and liver infarction occurring as a complication of acute bacterial endocarditis. Gastroenterology. 1975, 68:1602-1607. Accessed: September 24, 2017: https://www.gastrojournal.org/article/S0016-5085(75)80153-3/abstract?code=ygast-site. 10.1016/S0016-5085(75)80153-3

29. Selim K, Kaplowitz N: Hepatotoxicity of psychotropic drugs. Hepatology. 1999, 29:1347-1351. Accessed: May 10, 2017: https://aasldpubs.onlinelibrary.wiley.com/doi/abs/10.1002/hep.510290535. 10.1002/hep.510290535

30. Watson A, Williams R: Anoxic hepatic and intestinal injury from carbon monoxide poisoning . Br Med J. 1984, 289:1113. Accessed: September 24, 2017: https://www.ncbi.nlm.nih.gov/pmc/articles/PMC1443239/. 10.1136/bmi.289.6452.1113

31. Bernal W, Wendon J: Acute liver failure. N Engl J Med. 2013, 369:2525-2534. Accessed: May 10, 2017: https://www.nejm.org/doi/full/10.1056/nejmra1208937. 10.1056/NEJMra1208937

32. Lucey MR, Mathurin P, Morgan TR: Alcoholic hepatitis. N Engl J Med. 2009, 360:2758-2769. Accessed: September 24, 2017: https://www.nejm.org/doi/full/10.1056/NEJMra0805786. 10.1056/NEJMra0805786

33. Fuhrmann V, Kneidinger N, Herkner H, et al.: Hypoxic hepatitis: underlying conditions and risk factors for mortality in critically ill patients. Int Care Med. 2009, 35:1397-1405. Accessed: March 24, 2018: https://link.springer.com/article/10.1007\%2Fs00134-009-1508-2. 10.1007/s00134-009-1508-2

34. Stoller JK: Acute exacerbations of chronic obstructive pulmonary disease . N Engl J Med. 2002, 346:988-994. Accessed: March 24, 2018: https://www.nejm.org/doi/full/10.1056/NEJMcp012477. 10.1056/NEJMcp012477

35. Galiè N, Humbert M, Vachiery JL, et al.: 2015 ESC/ERS guidelines for the diagnosis and treatment of pulmonary hypertension. Eur Heart J. 2016, 37:67-119. Accessed: March 24, 2018: https://academic.oup.com/eurheartj/article/37/1/67/2887599. 10.1093/eurheartj/ehv317 